\title{
Remembering Jerry Marsden
}

\author{
T. Ratiu* \\ Ecole Polytechnique Fédérale de Lausanne \\ Chair of Geometric Analysis \\ and \\ Bernoulli Center \\ CH-1015 Lausanne, Switzerland \\ Received November 16, 2010
}

DOI: $10.1134 / \mathrm{S} 1560354710060018$

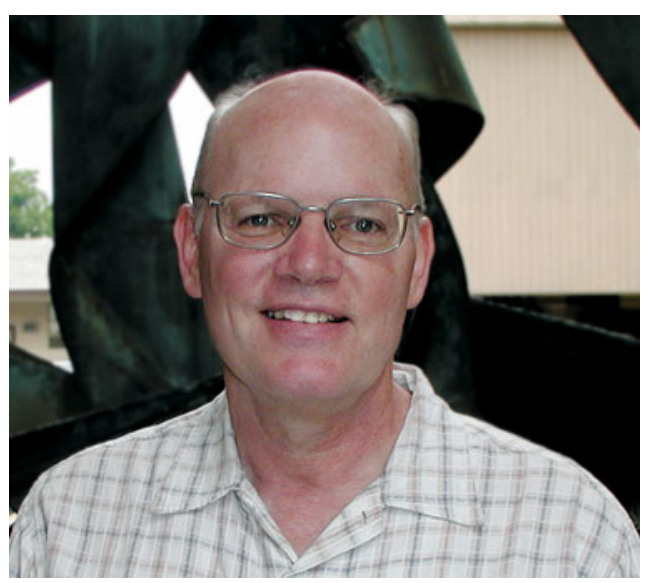

Jerrold E. Marsden, Jerry to his many friends, left us on the 21st of September, 2010, at the age of 68 . He leaves behind an applied mathematics school that he created by importing geometric and dynamical systems ideas into diverse applied areas such as numerical analysis, classical applied mathematics, theoretical physics, and engineering. He created strong links between pure and applied mathematics as well as between mathematics and various areas of physics, engineering, and chemistry. His ideas have influenced generations of researchers who have used geometry in their work.

Jerry Marsden was involved in the foundations of several areas. For example, the symplectic reduction theorem that he proved together with Alan Weinstein has become so fundamental in any research involving symmetry that it is today inconceivable to work in this field without using it. It has become one of the most cited theorems, often being referred to only by name without even giving a reference, since it is so basic. Jerry helped revive nonholonomic mechanics by introducing symmetry ideas. He used geometric ideas to study partial differential equations in hydrodynamics, elasticity, plasma physics, and relativity. He used symmetry techniques to investigate the nonlinear stability of relative equilibria and bifurcation phenomena associated to them. He has also linked aspects of control theory to symmetry. He used dynamical systems ideas in mission design and collaborated with researchers at the Jet Propulsion Laboratory in Pasadena to produce orbits for the Genesis mission. His ideas to use symplectic and variational methods in numerics greatly contributed to the recent spectacular development of geometric integration.

Jerry Marsden had the unusual talent to be able to communicate with scientists in other areas. He understood a question posed by a physicist, chemist, engineer, or biologist and then reformulated

${ }^{*}$ E-mail: tudor.ratiu@epfl.ch 
this vague and often open ended problem into a precise mathematical statement. Then he brought in the full power of pure mathematics to bear on it. For Jerry, pure mathematics was a branch of applied mathematics. His motivation was always a precise question that would shed light not only on the applied field where it came from, but also on the pure mathematics side where it would uncover structures and abstract theorems that always turned out to be relevant to other areas. His knowledge of mathematics and everything else surrounding it was encyclopedic, and so he was able to see links or the applicability of certain techniques between areas that were, a priori, very far apart. His power of abstraction was astounding. From a simple question in fluid dynamics, bifurcation theory, control, field theory, or numerics, he would, in a matter of minutes, outline a several years long research program encompassing difficult pure mathematics all the way to engineering implementations. Many of his associates considered him almost like an oracle, and an hour discussion with him could develop into years of serious collaboration. Jerry was also able to talk to everyone and explain mathematics at all levels. His door was always open, and anyone with a question or an idea was welcome. The only thing he insisted upon was commitment to the resolution of the problem, hard work, and serious scholarly research. He scrupulously gave credit to anyone who had ever worked on the problem under consideration and expected his collaborators to behave in the same way. His generosity in sharing ideas and helping out young scientists was legendary.

Jerry was also an unusually talented expositor, as can be seen from the many books he published that address audiences at all levels of mathematical sophistication. "Foundations of Mechanics", written jointly with Ralph Abraham, has practically created the field of modern geometric mechanics and is a classic. His book, "Mathematical Foundations of Elasticity", with Tom Hughes, has changed research directions in the mathematical theory of elasticity and has strongly influenced engineers. "A Mathematical Introduction to Fluid Mechanics", with Alexandre Chorin is one of most widely used texts by those interested in mathematical aspects of hydrodynamics. "Properties of Infinite Dimensional Hamiltonian Systems", written jointly with Paul Chernoff, and "Applications of Global Analysis in Mathematical Physics" remain to this day the standard references for anyone working on the rigorous functional analytic aspect of infinite dimensional conservative dynamics. Some of his books addressing the subject matter of various standard courses have become classics, are translated into several languages, and are used as the basis of undergraduate and graduate classes around the world. Due to this, many students flocked to him. He has supervised $49 \mathrm{Ph} . \mathrm{D}$. theses and a huge number of postdocs.

Jerry Marsden was born on August 17, 1942, in Ocean Falls, British Columbia, Canada, and graduated in 1965 from the University of Toronto. In 1968 he got his Ph.D. at Princeton University with Arthur Wightman as advisor and then went to the University of California at Berkeley. In 1995 he left for Caltech as professor in the program "Control and Dynamical Systems" of the Engineering Department where he remained till his death. He won numerous awards such as the the Norbert Wiener Prize (1990), the Humboldt Prize (1990-91, 1999), the Max Planck Research Award (2000), and the John von Neumann Prize (2005). He was given an honorary doctorate from the University of Surrey (2006). He was a Fellow of the American Academy of Arts and Sciences, of the Royal Societies of Canada and of the United Kingdom. He was the founder of the Fields Institute in Toronto, Canada, and a postdoctoral fellowship at this institution carries his name.

Jerry Marsden's expertise, openness, generosity, insight, and advice will be much missed by many scientists around the world. 